\title{
Osteocyte Remodeling of the Perilacunar and Pericanalicular Matrix
}

\author{
Hai Qing ${ }^{1,2}$, Lynda F. Bonewald ${ }^{1 *}$ \\ ${ }^{1}$ Department of Oral Biology, University of Missouri-Kansas City, Kansas City, USA \\ ${ }^{2}$ Department of Prosthodontics, West China College of Stomatology, Sichuan University, Chengdu, China
}

\section{Abstract}

Hai Qing, Lynda F. Bonewald. Osteocyte Remodeling of the Perilacunar and Pericanalicular Matrix. International Journal of Oral Science, 1(2): 59-65, 2009

With additional functions of osteocytes being identified, the concept that osteocytes are just "static lacunar-dwelling cells" is no longer accepted. We reviewed most of the relevant literature on osteocyte's function in the direct remodeling of the perilucunar matrix, discussing the advantages and disadvantages. Special attention was paid to how the negative researchers argue about the "osteocytic osteolysis" principle, and how the positive side addressed
\end{abstract}

the arguments. We also discussed the newly found data of osteocytic remodeling function from our group. With more biotechnology in hand, there is increased excitement in the prospect of now being able to answer the two important questions: do osteocytes have the capability to remove mineral from the perilacunar matrix and if so what are the molecular and cellular mechanisms? do osteocytes have the capability to deposit new mineral on the perilacunar matrix and if so what are the cellular and molecular mechanisms?

Keywords osteocyte, osteocytic resorption, remodeling, osteocytic osteolysis

Document code: A CLC number: Q21 Received Apr. 3, 2009; Revision accepted May 18, 2009

\section{Introduction}

Osteocytes are the most abundant and longestliving bone cells in the adult skeleton, being 10 times more abundant than osteoblasts with the potential to live as long as the host's life time (Parfitt, 1977). Osteocytes are regularly spaced throughout the mineralized matrix encased in cavelike structures called lacunae of 15-20 $\mu \mathrm{m}$ with numerous dendritic processes within small "tunnels" called canaliculi, approximately $250-300 \mathrm{~nm}$ in diameter (Donahue, 2000). In this manner, osteocyte "housing" forms an extensive lacunocanalicular network in the bone, allowing the osteocytes to maintain contact through their dendritic processes and communicate with each other and with cells on the bone surface such as lining cells, osteoclasts and osteoblasts (Figure 1).

The last decade has witnessed with greater fre- quency a rapid increase of interest in osteocytes, most likely because of new methods and state-ofthe-art technology responsible for new discoveries that have lead to breakthroughs in the study of osteocytes. Establishment of a cell line, MLO-Y4, captured the imagination of many investigators who now had access to a osteocyte-like cell (Kato et al., 1997) to examine osteocytic response to mechanical loading in the form of shear stress, osteocyte apoptosis, osteocyte signaling, and osteocyte communication through gap junctions and hemichannels (Bonewald, 2007). Breakthroughs were made in the identification of important markers for osteocyte differentiation which included E11/gp38, an early marker for embedding cells, Phex and dentin matrix protein 1, DMP1, important in metabolism, and Sost/sclerostin, a late marker for mature osteocytes (Bonewald, 2007).

Transgenic technology has also allowed advances 

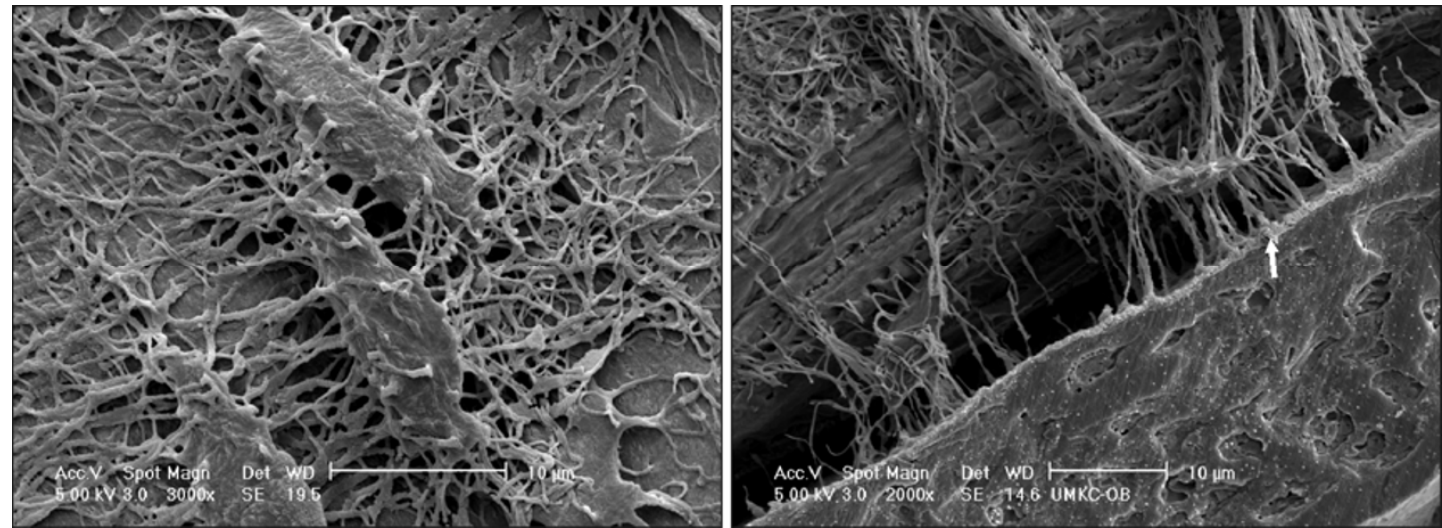

Figure 1 Osteocytes make contact with each other and cells on the surface

\begin{abstract}
The images are of acid-etched resin embedded murine bone visualized by scanning electron microscopy showing the high interconnectivity of the osteocyte lacuno-canalicular system. The left panel shows the complexity of the osteocyte lacuno-canalicular
\end{abstract} network and the right panel shows osteocyte canaliculi in contact with the surface of the bone (arrow)

in the study of osteocyte functions. The promoter for Dmp 1 has been used to generate animals with green fluorescent protein, GFP, reporter labeled osteocytes (Kalajzic et al., 2004) and to generate a Dmp1-Cre mouse to perform targeted deletion of genes in osteocytes (Feng et al., 2006). The use of this promoter has shown the importance of the PTH receptor in osteocytes to maintain and regulate bone mass (O'Brien et al., 2008; Divieti et al., 2005), and the importance of the Wnt/ $\beta$-catenin signaling pathway in osteocyte viability and maintenance of bone mass (kramer et al., 2008). However, it was the discovery of a marker for the late mature osteocyte (the gene is Sost and the protein is sclerostin), that began to interest the pharmaceutical industry. This osteocyte product appears to target the osteoblast to negatively regulate bone formation. Neutralizing antibody to sclerostin appears to increase bone mass and decrease bone loss, highlighting the osteocyte as a therapeutic target, something previously applied to the osteoclast and osteoblast (Li et al., 2008).

While new functions of osteocytes have been discovered such as their role in phosphate metabolism (Liu et al., 2006), previous functions proposed for osteocytes have been rediscovered. This review will focus on rediscovering an earlier function of osteocytes, that of being able to remove and replace their perilacunar matrix, referred to by Belanger as "osteocytic osteolysis" (Belanger et al., 1967). Again, discoveries of osteocyte function regarding removal of their perilacunar matrix, is attributable to new technology, in this case the use of new imaging technologies such as Raman spectroscopy, atomic force microscopy, and synchrotron technology.

\section{A "local bone remodeling" function for osteocytes}

The surface area of the osteocyte lacuno-canalicular system within bone is several orders of magnitude greater than the bone surface area that is directly remodeled by osteoblasts and osteoclasts (Marotti et al., 1995). Therefore osteocytes have access to an extremely large area and the removal of only a few angstroms of mineral per osteocyte would have significant effects on circulating, systemic ion levels.

This feature of the lacuno-canalicular system inspired bone researchers to hypothesize as early as 100 years ago that osteocytes might have the ability to directly mobilize the bone mineral from the inner lacunar surface. In 1910, von Recklinghausen described enlarged lacunae in patients with rickets or osteomalacia (Recklinghausen, 1910), which suggested to him that pericellular "digestion" was occurring around the osteocytes (Hellerteinberg, 1951). Interest in this area increased in the $1970 \mathrm{~s}$ as evidenced by the number of publications, however, this was followed by an obvious decrease from the 1980s to the present (Figure 2). We will discuss the rise and fall and the new rise in interest 

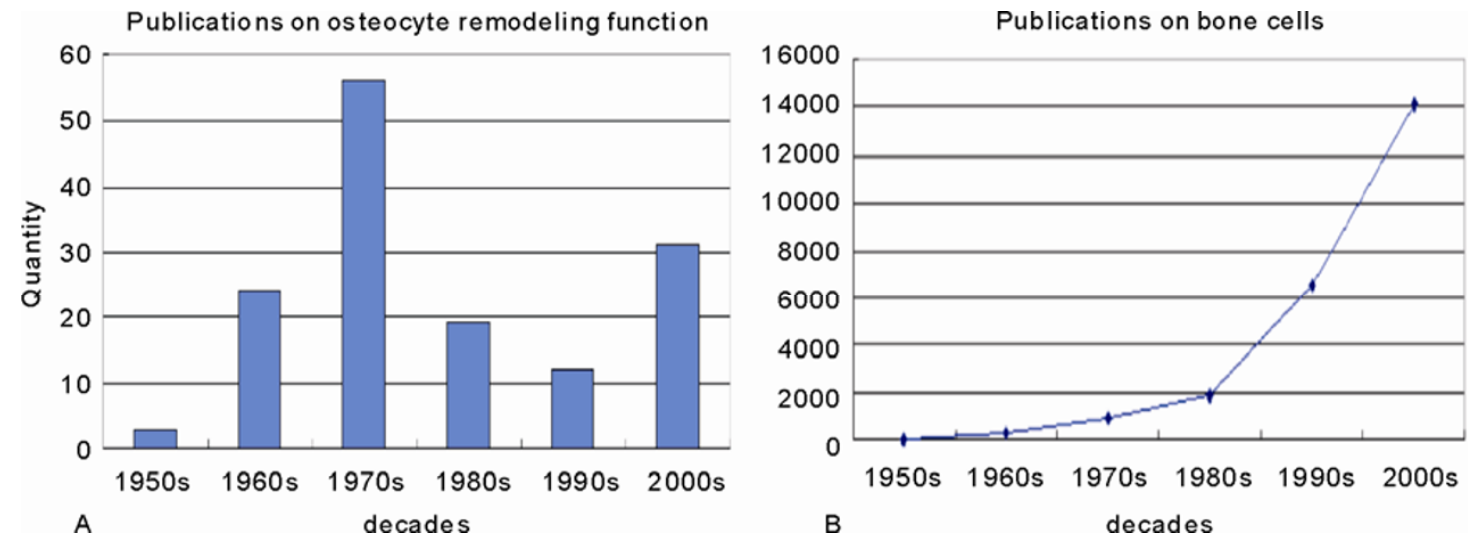

Figure 2 Summary of number of publications obtained from Pubmed

A: Using the keywords "osteocytic osteolysis", "osteocytic resorption", "periosteocytic osteolysis" or "osteoplasitic". B: Using the keywords "osteoblast", "osteoclast" or "osteocyte".

in this area. One must keep in mind that these early pioneers in osteocyte biology mainly had the tools of histology and histomorphometry. Their interpretations of their histology were insightful, but limitations left few means to prove a hypothesis. With our new technology, scientists are posed to test the hypotheses of these early pioneers.

\section{Enlargement of osteocyte lacunae}

In 1951, Heller-Steinberg showed that the areas located around lacunae and sometimes around canaliculi were positive using periodic acid-Schiff (PAS) stain in rat bone treated with parathyroid extract (Heller-Steinberg, 1951). PAS stain can detect polysaccharides and glycoproteins which are normally detected in unmineralized bone matrix or osteoid. Ruth and colleagues found basophilic matrix around osteocytes in bone from lactating rats that had been fed a calcium-free diet (Ruth, 1961). A basophilic matrix suggests what was referred to as "acidic ground material" in the matrix was mainly composed of glycoproteins. These early findings suggested that mineral removal might be taking place around the osteocytes in these animals. However, these investigators were unable to show a direct correlation between matrix staining (PAS, basophilic or toluidine blue) and mineral removal or bone resorption. It was indicated that if the cell forms bone matrix first before incorporation of mineral, this technique will give false results regarding mineral removal. Conversely, if the osteocyte removes both the mineral and the matrix, this form of matrix staining cannot detect this type of matrix removal.

Baud was credited in 1962 to be the first person to describe rough borders of lacunar walls of osteocytes using electron microscopy again suggesting the osteocytic removal of perilacunar matrix (Baud, 1962). Later, he used microradiographs to show that parathyroid hormone can induce "osteocytic resorption" based on enlarged lacunae (Baud, 1968a; Baud, 1968b). Belanger, around this same time was also investigating this phenomena and coined the term "osteocytic osteolysis" and published a series of papers suggesting that either parathyroid hormone or low-calcium diet can induce this function in osteocytes (Belanger and Robichon, 1964; Belanger and Drouin, 1966; Belanger, 1969). In renal osteodystrophy, a histologic investigation found a significant increase in the number of enlarged and irregular lacunae in uremic subjects (Bonucci and Gherardi, 1975; Bonucci et al., 1976). In 1977, Iagodovskii et al. sent rats into space for a 22-day space flight. They found by light and electron microscopy "wide osteocyte lacunae that could be associated with perilacunar osteolysis" (Iagodovskii et al., 1977). This work found a relationship between "osteocytic osteolysis" and microgravity. Additionally, "osteocytic osteolysis" was found in the alveolar bone of hibernating ground squirrels (Haller and Zimny, 1977). In an attempt to uncover or determine the 
mechanism whereby osteocytes might remove their matrix Belanger in 1963 reported the presence of protease "mainly over mature osteocytes" (Belanger and Migicovsky, 1963). Later, he reported the presence of lysosomes in the large, mature osteocytes and the stimulating effects of parathyroid hormone on these vesicles (Belanger, 1969).

\section{What happened to "osteocytic osteolysis"?}

Even though the aforementioned early investigations tried to establish the function of osteocytes in bone removal in terms of "resorption", there was not a general acceptance of the osteocytic osteolysis concept. The reasons were (1)combination of this concept "osteocytic osteolysis" with a second concept "bone flow" that proved incorrect; (2) assuming that the removal of bone matrix by osteocytes would occur similarly to osteoclastic bone resorption; (3)problems with sample preparation; and (4)lack of additional methods to test the concept. When Krook and Belanger developed their "bone flow-osteocytic osteolysis" theory in 1970 (Krook et al., 1970), considerable controversy was generated. In 1977, Parfitt published a paper disproving the "bone-flow theory" which stated that osteocytes rather than osteoclasts resorb the bone internally and then bone flowed into the osteocyte-resorbed space. This "bone-flow theory" is now known to be incorrect, but Parfitt also mentioned osteocytic osteolysis as an artifact by saying that the procedure might artificially produce the enlargement of lacunae (Parfitt, 1977). This publication, from a highly respected bone biologist, was partially responsible for the decrease of interest in "osteocytic osteolysis" after the 1970s (Figure 2). In addition, van der Plas and co-workers assumed that osteocytes would form resorption lacunae or pits similar to those formed by osteoclasts. When isolated avian osteocytes did not form resorption lacunae when cultured on sperm whale dentin, the concept of osteocyte resorption was discredited (van der Plas et al., 1994). The irregular morphology, variability in spacing, and lack of consistent orientation especially in trabecular bone of osteocyte lacunae created technical challenges when using two dimensional measurements. These inconsistencies appeared to confound two dimensional measurements that were used to predict three dimensional volumes. No consensus on a criterion for the morphological evaluation has been agreed upon. Also similar changes in lacunae can also be found in the younger osteocytes, therefore these enlarged lacunae could be due to defective mineralization of the periosteocytic matrix by the embedding osteoid osteocyte (Boyde and Jones, 1979; Parfitt, 1977).

However, three papers need to be noted in this "cold period" which used different approaches to provide more supportive evidence for osteocytic resorption. Alcobendas used microradiographs to show that osteocyte lacunae of breeding female or hibernating snakes are significantly enlarged compared to corresponding controls (virgin and non-hibernating snakes). In the snake model, the remodeling of the bone tissue does not occur throughout the life of the animal. Therefore the enlarged lacunae should come from the embedded mature osteocytes and not young, newly forming osteocytes (Alcobendas et al., 1991). Nakano and co-workers used in situ hybridization for gene expression and immunostaining to show tartrate resistant acid phosphatase (TRAP) thought to be an osteoclast specific marker, in osteocytes. In situ hybridization eliminated the possibility that TRAP is diffused from the nearby osteoclast (Nakano et al., 2004), a criticism of the Baylink work in 1969 (Wergedal and Baylink, 1969). Lane and colleagues compared changes in lacunar size in 6-month-old male glucocorticoid-treated and female ovariectomized mice to their respective controls and showed that lacunar size was increased in those two treated groups, significantly in the first group (Lane et al., 2006). They used three dimensional reconstruction methods to obtain lacunar volume, which is a theoretically more reliable measurement to eliminate the confounding osteocyte-orientation factor.

\section{Osteocytic replacement of perilacunar matrix/mineral}

Whereas before the 1970s there was a considerable number of manuscripts reporting "osteocytic osteolysis", there were very few reporting osteocytic replacement of this previously removed matrix. In 1971, Baylink used tetracycline labeling 
to show tetracycline binding to the perilacunar matrix, which led him to suggest that osteocytes have the ability to form bone. He also reported that compared with osteoblasts which have a higher bone-forming rate, osteocytes do not play a major role in regulating serum calcium (Baylink and Wergedal, 1971). Later, Zallone used the egglaying hen and several methods including autoradiography and tetracycline labeling to show that at least $20 \%$ of the osteocytes are active in bone formation, with relatively higher values in the metaphysis than in the diaphysis (Zambonin Zallone et al., 1982; Zambonin Zallone et al., 1983). Currently, the molecular mechanisms responsible for perilacunar matrix replacement are not known but are speculated to be similar to the osteoblast. It would make sense that the osteocyte could revive its previous memory as a matrix producing osteoblast, but this remains to be proven.

\section{Implications for osteocyte remodeling of their local environment}

Our group has found that lacunae are significantly enlarged during lactation in both cortical and trabecular bone in tibiae and lumber vertebrae compared with virgin and post-weaning group, and this change is correlated with TRAP expression (Qing et al., 2008). This study shows that healthy osteocytes can remove and replace their perilacunar matrix and potentially play a role in mineral homeostasis during a calcium-demanding condition such as lactation. However, in 1985, Mercer et al. reported that rat osteocytes do not resorb bone during lactation based on toluidine blue staining (Mercer et al., 1985). However, it should be noted that toluidine blue stains proteoglycans and glycosaminoglycans (Shepard and Mitchell, 1976; Zambonin Zallone et al., 1983), therefore enlargement of the lacunae might not be detected. Secondly, the metachromatically toluidine blue stained lacunae may undergo osteocytic resorption (Zambonin Zallone et al., 1983). Lastly, the resolution of this measurement may not be sufficient to reveal significant differences.

Nicolella reported that the osteocyte lacuna acts as a strain concentrator effectively amplifying the macroscopic strain applied to the whole bone and this amplification factor is a function of the local perilacuna bone tissue material properties (Nicolella et al., 2006; Nicolella et al., 2008). If mechanical loading is a major regulator of osteocyte function, what does this mean for the osteocyte that is hormonally regulated to remove and replace its matrix? Are the effects of mechanical loading ignored or overridden in the case of enlarged lacunae with lactation? Are the effects of mechanical loading diminished with the enlargement of lacunae with hyperparathyroidism? Do changes in lacunar size with aging affect response to mechanical load? These are questions that remain to be answered.

Additionally, the canalicular surface area is 10 times larger than lacunar surface area, which means that potentially osteocyte dentrites could also be involved in removal and replacement of bone matrix and mineral. However, to date, there is no reliable method to investigate this tiny (about 250-300 nm) and irregular structure of the canaliculi. There is the potential for synchrotron technology to be useful in this determination.

\section{Summary}

Since additional functions of osteocytes are being identified, the concept that osteocytes are just "static lacunar-dwelling cells" is no longer accepted. More and more bone biologists would speculate that osteocytes can be involved in the local bone matrix remodeling to mobilize mineral ions, but sufficient convincing evidence has still not been presented. Regarding the osteocyte local remodeling function, there are two questions needed to be addressed; Do osteocytes have the capability to remove mineral from the perilacunar matrix and if so what is the molecular and cellular mechanisms? Do osteocytes have the capability to deposit new mineral on the perilacunar matrix and if so what is the cellular and molecular mechanisms? There is increased excitement with the prospect of now being able to answer these questions.

\section{Acknowledgements}

The authors' work is supported in part by the 
National Institutes of Health AR-46798.

\section{References}

Alcobendas M, Baud CA, Castanet J (1991). Structural changes of the periosteocytic area in Vipera aspis (L.) (Ophidia, Viperidae) bone tissue in various physiological conditions. Calcif Tissue Int, 49(1): 53-57.

Baud CA (1962). Morphology and inframicroscopic structure of osteocytes. Acta Anat (Basel), 51: 209-225.

Baud CA (1968a). Structure and functions of osteocytes in normal conditions and under the influence of parathyroid extract. Schweiz Med Wochenschr, 98(19): 717-720.

Baud CA (1968b). Submicroscopic structure and functional aspects of the osteocyte. Clin Orthop Relat Res, 56: 227-236.

Baylink DJ, Wergedal JE (1971). Bone formation by osteocytes. Am J Physiol, 221(3): 669-678.

Belanger LF (1969). Osteocytic osteolysis. Calcif Tissue Res, 4(1): 1-12.

Belanger LF, Belanger C, Semba T (1967). Technical approaches leading to the concept of osteocytic osteolysis. Clin Orthop Relat Res, 54: 187-196.

Belanger LF, Drouin P (1966). Osteolysis in the frog. The effects of parathormone. Can J Physiol Pharmacol, 44(6): 919-922.

Belanger LF, Migicovsky BB (1963). Histochemical evidence of proteolysis in bone: The influence of parathroid hormone. J Histochem Cytochem, 11: 734737.

Belanger LF, Robichon J (1964). Parathormone-induced osteolysis in dogs. A microradiographic and alpharadiographic survey. J Bone Joint Surg Am, 46: 1008012.

Bonewald LF (2007). Osteocytes. In: Osteoporosis, 3 ed. Marcus R, Feldman D, Nelson DA, Rosen CJ, eds. Burlington: Elsevier Academic Press, pp. 169-190.

Bonucci E, Gherardi G (1975). Histochemical and electron microscopy investigations on medullary bone. Cell Tissue Res, 163(1): 81-97.

Bonucci E, Gherardi G, Faraggiana T (1976). Bone changes in hemodialyzed uremic subjects. Comparative light and electron microscope investigations. Virchows Arch A Pathol Anat Histol, 371(3): 183-198.

Boyde A, Jones SJ (1979). Estimation of the size of resorption lacunae in mammalian calcified tissues using SEM stereophotogrammetry. Scan Electron Microsc, 2(2): 393-402.
Divieti P, Geller AI, Suliman G, Juppner H, Bringhurst FR (2005). Receptors specific for the carboxyl-terminal region of parathyroid hormone on bone-derived cells: determinants of ligand binding and bioactivity. Endocrinology, 146(4): 1863-1870.

Donahue HJ (2000). Gap junctions and biophysical regulation of bone cell differentiation. Bone, 26(5): 417-422.

Feng JQ, Ward LM, Liu S, Lu Y, Xie Y, Yuan B, et al. (2006). Loss of DMP1 causes rickets and osteomalacia and identifies a role for osteocytes in mineral metabolism. Nature Genetics, 38(11): 1310-1315.

Haller AC, Zimny ML (1977). Effects of hibernation on interradicular alveolar bone. J Dent Res, 56(12): 1552557.

Heller-Steinberg M (1951). Ground substance, bone salts, and cellular activity in bone formation and destruction. Am.J.Anat. 89(3): 347-379.

Iagodovskii VS, Triftanidi LA, Gorokhova GP (1977). Effect of space flight on rat skeletal bones (an optical light and electron microscopic study). Kosm Biol Aviakosm Med, 11(1): 14-20.

Kalajzic I, Braut A, Guo D, Jiang X, Kronenberg MS, Mina M, et al. (2004). Dentin matrix protein 1 expression during osteoblastic differentiation, generation of an osteocyte GFP-transgene. Bone, 35(1): 74-82.

Kato Y, Windle JJ, Koop BA, Mundy GR, Bonewald LF (1997). Establishment of an osteocyte-like cell line, MLO-Y4. J Bone Miner Res, 12(12): 2014-2023.

Kramer I, Halleux C, Brander Weber P, Feng JQ, Boisclair J, Keller H, et al. (2008). Osteocyte-specific ablation of canonical Wnt signaling induces severe osteoporosis. ASBMR 30th anual meeting, 23: s12.

Krook L, Belanger LF, Henrikson PA, Lutwak L, Sheffy BE (1970). Bone flow. Rev Can Biol, 29(2): 157-167.

Lane NE, Yao W, Balooch M, Nalla RK, Balooch G, Habelitz S, et al. (2006). Glucocorticoid-treated mice have localized changes in trabecular bone material properties and osteocyte lacunar size that are not observed in placebo-treated or estrogen-deficient mice. J Bone Miner Res, 21(3): 466-476.

Li X, Ominsky MS, Warmington KS, Morony S, Gong J, Cao J, et al. (2008). Sclerostin antibody treatment increases bone formation, bone mass and bone strength in a rat model of postmenopausal osteoporosis. J Bone Miner Res, 24(4): 578-588.

Liu S, Tang W, Zhou J, Stubbs JR, Luo Q, Pi M, et al. (2006). Fibroblast growth factor 23 is a counter- 
regulatory phosphaturic hormone for vitamin D. $J \mathrm{Am}$ Soc Nephrol, 17(5): 1305-1315.

Marotti G, Ferretti M, Remaggi F, Palumbo C (1995). Quantitative evaluation on osteocyte canalicular density in human secondary osteons. Bone, 16(1): 125-128.

Mercer RR, Crenshaw MA, Rasmussen P. (1985). The role of osteocytes in bone resorption during lactation: morphometric observations calcium deficiency, pregnancy, and lactation in rats. Microscopic and microradioaphic observations on bones. Bone, 6(4): 269274.

Nakano Y, Toyosawa S, Takano Y (2004). Eccentric localization of osteocytes expressing enzymatic activities, protein, and mRNA signals for type 5 tartrateresistant acid phosphatase (TRAP). J Histochem Cytochem, 52(11): 1475-1482.

Nicolella DP, Feng JQ, Moravits DE, Bonivitch AR, Wang Y, Dusecich V, et al. (2008). Effects of nanomechanical bone tissue properties on bone tissue strain: implications for osteocyte mechanotransduction. $J$ Musculoskelet Neuronal Interact, 8(4): 330-331.

Nicolella DP, Moravits DE, Gale AM, Bonewald LF, Lankford J (2006). Osteocyte lacunae tissue strain in cortical bone. J Biomech, 39(9): 1735-1743.

O'Brien CA, Plotkin LI, Galli C, Goellner JJ, Gortazar AR, Allen MR, et al. (2008). Control of bone mass and remodeling by $\mathrm{PTH}$ receptor signaling in osteocytes. PLoS ONE, 3(8): e2942.
Parfitt AM (1977). The cellular basis of bone turnover and bone loss: a rebuttal of the osteocytic resorption-bone flow theory. Clin Orthop Relat Res, 127: 236-247.

Qing H, Ardeshirpour L, Dusecich V, Dallas M, Wysolmerski JJ, Bonewald LF (2008). Osteocytic perilacunar remodeling as a significant source of calcium during lactation. ASBMR 30th anual meeting, 23: s401.

Recklinghausen FV (1910). Untersuchungen uber Rachitis und Osteomalacia. Jena: Gustav Fischer.

Ruth EB (1961). Basophilic islands in osseous tissue and their relation to resorption. Anat Rec, 140: 307-320.

van der Plas A, Aarden EM, Feijen JH, de Boer AH, Wiltink A, Alblas MJ, et al. (1994). Characteristics and properties of osteocytes in culture. J Bone Miner Res, 9(11): 1697-1704.

Wergedal JE, Baylink DJ (1969). Distribution of acid and alkaline phosphatase activity in undemineralized sections of the rat tibial diaphysis. $J$ Histochem Cytochem, 17(12): 799-806.

Zambonin Zallone AZ, Teti A, Nico B, Primavera MV (1982). Osteoplastic activity of mature osteocytes evaluated by H-proline incorporation. Basic Appl Histochem, 26(1): 65-67.

Zambonin Zallone AZ, Teti A, Primavera MV, Pace G. (1983). Mature osteocytes behaviour in a repletion period: the occurrence of osteoplastic activity. Basic Appl Histochem, 27(3): 191-204.

*Corresponding author: Lynda F. Bonewald

Address: Department of Oral Biology, University of Missouri-Kansas City, Kansas City, MO 64108, USA

Tel: 018162356296 E-mail: Bonewaldl@umkc.edu 